OPEN ACCESS

Edited by:

Alberto Biondi,

Catholic University of the Sacred

Heart, Italy

Reviewed by:

Min Ma,

Central South University, China

Stefano Rausei,

ASST Valle Olona, Italy

${ }^{*}$ Correspondence: Shi Chen

chensh47@mail.sysu.edu.cn Jun-Sheng Peng

pengjsh@mail.sysu.edu.cn

${ }^{\dagger}$ These authors have contributed equally to this work

Specialty section:

This article was submitted to Gastrointestinal Cancers,

a section of the journal

Frontiers in Oncology

Received: 10 August 2020

Accepted: 06 April 2021

Published: 30 April 2021

Citation:

Chen Y-H, Lu J, Nie R-C

Liu D, Liu A-H, Deng Z-J, Chen X-J, Xiang $J$, Chen $Y-B$, Huang $C-M$,

Chen $S$ and Peng J-S (2021)

Retrieval of 30 Lymph Nodes is

Mandatory for Selected Stage II

Gastric Cancer Patients.

Front. Oncol. 11:593470.

doi: 10.3389/fonc. 2021.593470

\section{Retrieval of 30 Lymph Nodes Is Mandatory for Selected Stage II Gastric Cancer Patients}

\author{
Yong-He Chen ${ }^{1,2,3 \dagger}$, Jun $\mathrm{Lu}^{4 \dagger}$, Run-Cong Nie ${ }^{5 \dagger}$, Dan Liu ${ }^{6}$, Ai-Hong Liu ${ }^{1,2,3}$, \\ Zi-Jian Deng ${ }^{1,2,3}$, Xi-Jie Chen ${ }^{1,2,3}$, Jun Xiang ${ }^{1,2,3}$, Ying-Bo Chen ${ }^{5}$, Chang-Ming Huang ${ }^{4}$, \\ Shi Chen ${ }^{1,2,3^{*}}$ and Jun-Sheng Peng ${ }^{1,2,3^{*}}$ \\ ${ }^{1}$ Department of Gastrointestinal Surgery, The Sixth Affiliated Hospital, Sun Yat-Sen University, Guangzhou, China, \\ 2 Department of Surgery, Guangdong Institute of Gastroenterology, Guangzhou, China, ${ }^{3}$ Department of Surgery, Guangdong \\ Provincial Key Laboratory of Colorectal and Pelvic Floor Diseases, Guangzhou, China, ${ }^{4}$ Department of Gastric Surgery, \\ Fujian Medical University Union Hospital, Fuzhou, China, ${ }^{5}$ Department of Gastric Surgery, Sun Yat-sen University Cancer \\ Center, State Key Laboratory of Oncology in South China, Collaborative Innovation Center for Cancer Medicine, Guangzhou, \\ China, ${ }^{6}$ Department of Laboratory Science, The Second Affiliated Hospital, Guangzhou University of Chinese Medicine, \\ Guangzhou, China
}

Background: According to the 8th edition AJCC staging manual, a least of 16 lymph nodes retrieval (LNRs) is the minimal requirement for lymph nodes (LNs) dissection of gastric cancer surgery. Previous studies have shown that increasing the number of LNRs $(\geq 30)$ prolongs survival for selected patients. However, the necessity of retrieving 30 or more LN for stage II gastric cancer patients is still under debate.

Aim: This study aims to explore the impact of retrieving 30 or more lymph nodes on the survival of stage II cancer patients.

Methods: A total of 1,177 patients diagnosed with stage II gastric cancer were enrolled in this study. The clinicopathological parameters and the impact of different LNRs $(<30$ or 230) and positive lymph node ratio (NR) on overall survival (OS) were retrospectively analyzed.

Results: The mean number of LNRs was $34 \pm 15$. A total of $44 \%(518 / 1,177)$ of patients had an LNRs $<30$, while $56 \%(659 / 1,177)$ of patients had an LNRs $\geq 30$. The 5 -year survival rate was $81 \%$ for all patients, $76 \%$ for the LNRs $<30$ group, and $86 \%$ for LNRs $\geq 30$ group, respectively $(P=0.003)$. The survival benefit of retrieving 30 lymph nodes was significant in certain subgroups: age $>60$ years/male/underwent total gastrectomy/stage IIB. For N+ patients, higher NR was significantly correlated with poor survival.

Conclusion: The survival benefit of retrieving $30 \mathrm{LNs}$ varies in different subgroups. An LNRs of 30 is mandatory for selected stage II gastric cancer patients.

Keywords: stage II, gastric cancer, prognosis, Chinese people, lymph node retrieval 


\section{INTRODUCTION}

Gastric cancer is the fourth most common malignant tumor in the world and is one of the most common causes of cancerrelated death (1). Lymphatic metastasis is a major metastatic pathway for gastric cancer. Extended lymph node dissection is an important part of radical gastrectomy. According to the $8^{\text {th }}$ edition of the AJCC staging manual, the retrieval of at least 16 lymph nodes is the minimal requirement for lymph node dissection, and retrieval of 30 lymph nodes is more desirable (2). Studies have found that an increase in the number of lymph nodes retrieved is associated with prolonged survival (3-10) because more lymph nodes retrieved may indicate more extended lymph node dissection and may help avoid tumor migration to a lower stage than the actual stage. Thus, some studies have proposed an argument that the minimal number of LNRs should be improved to a higher standard. However, there are also some studies stating that an increase in the LNRs only benefits certain groups of patients. Macalindong et al. found in a high-volume gastric cancer data set that retrieving 30 or more LNs did not influence the survival of stage II GC patients (4). Vuong et al. also found that retrieving 30 or more LNs only benefited those with a more advanced N stage (11). The attempt to harvest more LNs may bring forth more postoperative complications $(6,12)$. To date, the necessity of retrieving 30 or more LNs for stage II gastric cancer patients is still debatable.

In our opinion, the minimal requirement for lymph node retrieval should be individualized according to the characteristics of the patients and the tumor.

This study aims to explore the impact of retrieving 30 or more lymph nodes on the survival of selected stage II cancer patients.

\section{MATERIALS AND METHODS}

\section{Study Population and Data Collection}

A total of 1,177 patients who received curative surgery from April 2008 to May 2017 were identified from the gastrointestinal malignancy cancer database of The Sixth Affiliated Hospital of Sun Yat-Sen University, Fujian Medical University Union Hospital, and Sun Yat-Sen University Cancer Center.

The inclusion criteria were as follows: (i) patients with histologically confirmed adenocarcinoma of the stomach or esophagogastric junction; (ii) patients who underwent gastrectomy with standardized D2 lymphadenectomy; and (iii) a post-surgery pathological stage of II according to the $8^{\text {th }}$ edition of the AJCC staging manual. The exclusion criteria were as follows: (i) patients with insufficient information; (ii) patients who received preoperative neoadjuvant therapy.

After initial screening, a total of 1,177 patients were included in this study. All available clinical information was retrieved from the database, including general patient demographics, tumor location, differentiation, tumor size, resection extent, LNRs, tumor stage, and survival. The patient information is listed in Table 1. The primary endpoint of this study is overall survival. Post-operative
Surveillance followed the recommendation of the National Comprehensive Cancer Network guideline (13). Follow-up visits for all three institutions generally consist of clinic visits every 6 months for the first 2 years and annually up to 5 years. Most routine patient follow-up appointments include a physical examination, laboratory tests, chest-abdominal computed tomography scan, and an annual endoscopic examination. In all the three institutions involved in this study, patients' follow-up was conducted by the staff of the follow-up offices. After the surgery, the follow-up office generally contacts the patients or the patients' families every 6 months, by telephone calls or mails, to gather information on the patients' condition and survival. This retrospective study was approved by the Institutional Review Board of all three centers.

\section{Surgery}

All patients received total or subtotal gastrectomy with D2 lymphadenectomy following the guidelines of the Japanese Gastric Cancer Association (14) (open or laparoscopic surgery depending on the surgeon's preference). A thorough examination of the abdominal cavity was routinely performed to determine the status of peritoneal metastasis. Peritoneal washing cytology tests were not routinely conducted. The extent of gastric resection was determined by the anatomical location of the tumor. Proximal or total gastrectomy with esophagogastrostomy or Roux-en-Y esophagojejunostomy reconstruction was performed for tumors located in the upper or middle third part of the stomach; distal subtotal gastrectomy with Billroth I, Billroth II, or Roux-en-Y gastrojejunostomy reconstruction was performed for tumors located in the distal third part of the stomach.

\section{Specimens Assessment}

Retrieval of lymph nodes from the gross specimens was by manual method, which means operators identified suspicious lymph nodes by sight and palpation and reconfirmed them under microscopic view. Station labeling was determined according to the anatomical sites and their relationship to the perigastric vessels. This procedure was performed by the surgeons on the fresh specimens instantly after surgical resection. Lymph nodes removed individually during surgery are labeled for stations by the surgeons and inspected separately. Pathological staging was determined according to the AJCC TNM staging system (2). In the final analysis, the patients were divided into two subgroups: patients with less than 30 lymph nodes retrieved (LNRs $<30$ group) and patients with 30 or more lymph nodes retrieved (LNRs $\geq 30$ group).

\section{Data Analysis}

The clinicopathological characteristics were compared using $\chi 2$ tests for categorical variables and analysis of variance (ANOVA) for continuous variables, as appropriate. Patients were divided into different subgroups according to the clinicopathological features (age, sex, the extent of gastrectomy, tumor location, 
TABLE 1 | Patient characteristics and $P$ value of univariate analysis.

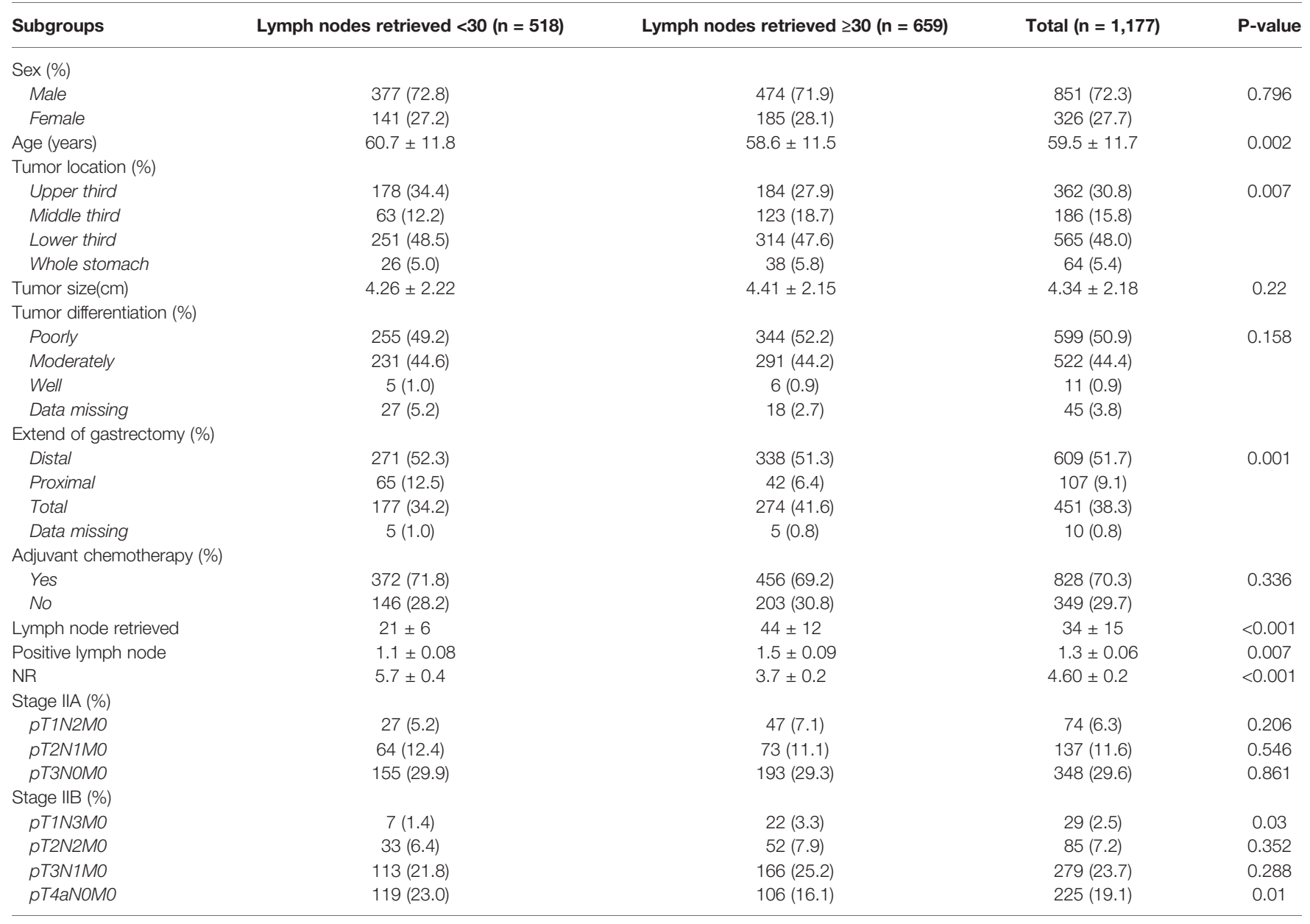

tumor differentiation, $\mathrm{T}$ stage, and $\mathrm{N}$ stage). For each subgroup, the Kaplan-Meier method and log-rank test were used to assess the survival impact of different lymph node retrievals (LNRs $<30$ versus LNRs $\geq 30$ ). The hazard ratio (HR) and the $95 \%$ confidence interval (95\% CI) were calculated by the Cox proportional hazards model, upon which a forest plot was built to visualize the impact on survival. A $\mathrm{P}$ value $<0.05$ was considered statistically significant. Statistical analysis was performed with SPSS version 25.0 for Windows (IBM, Armonk, NY, USA).

\section{RESULTS}

\section{Patient Characteristics}

A total of 1,177 patients were enrolled in this study. The majority of patients were male. The patients' median age at diagnosis was 59 years (range, 16 to 91 years). Nearly half of the tumors were located in the distal part of the stomach, also half of the tumors were poorly differentiated, other details are depicted in Table 1. The spectrum of the specific pTNM stage is shown in Figure 1, different pTN stages were roughly balanced in the LNRs $\geq 30$ and $<30$ subgroups.

\section{Lymph Node Retrieval}

The mean number of LNRs was $34 \pm 15$, with a range from 3 to 101. Numbers of patients with LNRs $<30(518 / 1,177,44 \%)$ and LNRs $\geq 30(659 / 1,177,56 \%)$ were roughly balanced. The distribution of LNRs and numbers of positive lymph nodes are shown in Figures 2, 3, respectively. The numbers of positive lymph nodes showed a tendency of increasing as the LNRs increased. In the LNRs $\geq 30$ subgroups, with the increase in the numbers of total LNRs, the numbers of positive lymph nodes were slightly higher, but the positive lymph nodes ratio (NR) was significantly lower than the LNRs $<30$ subgroup.

\section{Survival Analyses}

Up to January 2020, in a median follow-up time of 44 months, a total of 168 tumor-related death events were observed in the 1,177 patients. The 5 -year survival rate was $81 \%$ for all patients, $76 \%$ for the LNRs $<30$ group, and $86 \%$ for LNRs $\geq 30$ group, respectively $(\mathrm{P}=0.003)$. The survival and hazard ratios adjusted by subgroups are depicted in Figures 4, 5. Although overall speaking, survival was significantly improved in the LNRs $\geq 30$ group, it was clear that the survival benefit of retrieving $30 \mathrm{LNs}$ varied in different subgroups. Patients aged $>60$ years $/$ male/who underwent total gastrectomy/stage IIB tended to benefit from an 


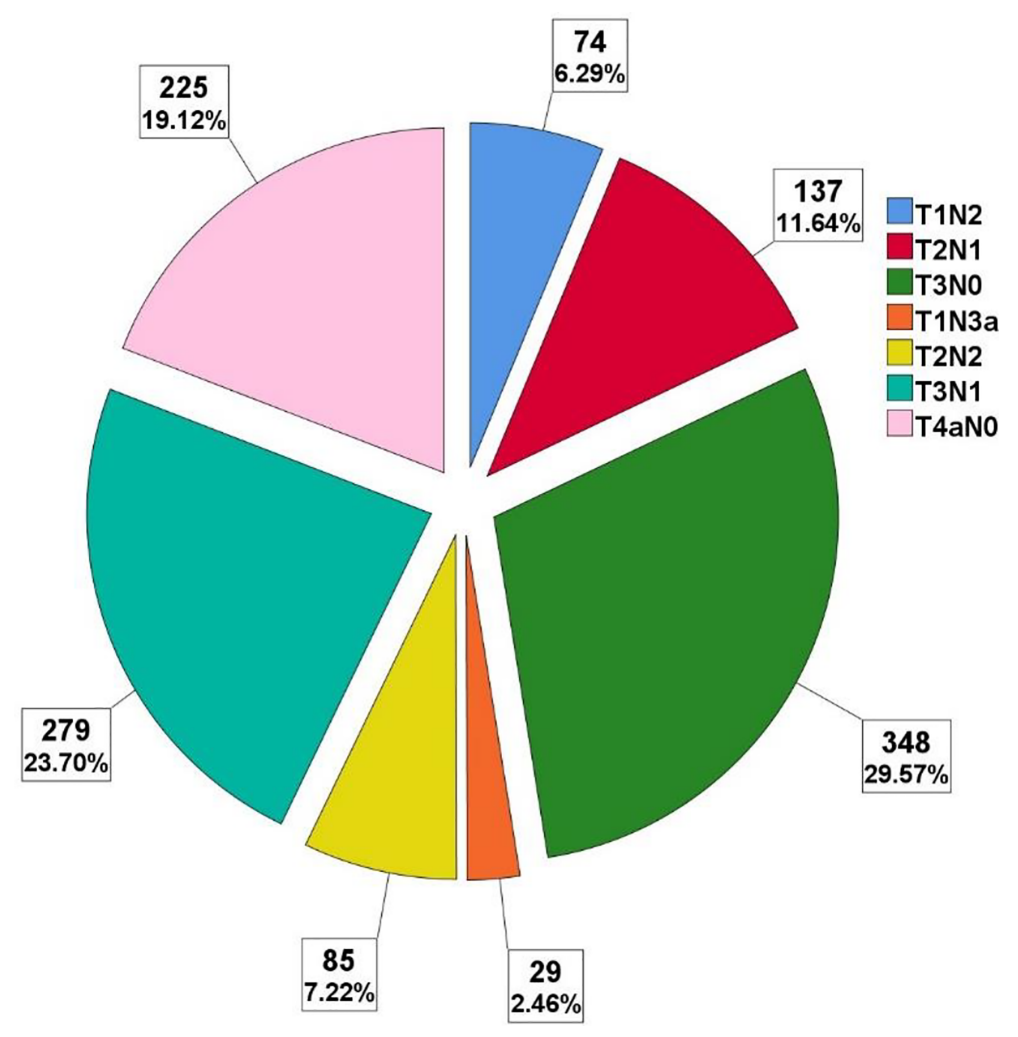

FIGURE 1 | Cases and proportion of different pTN stages.

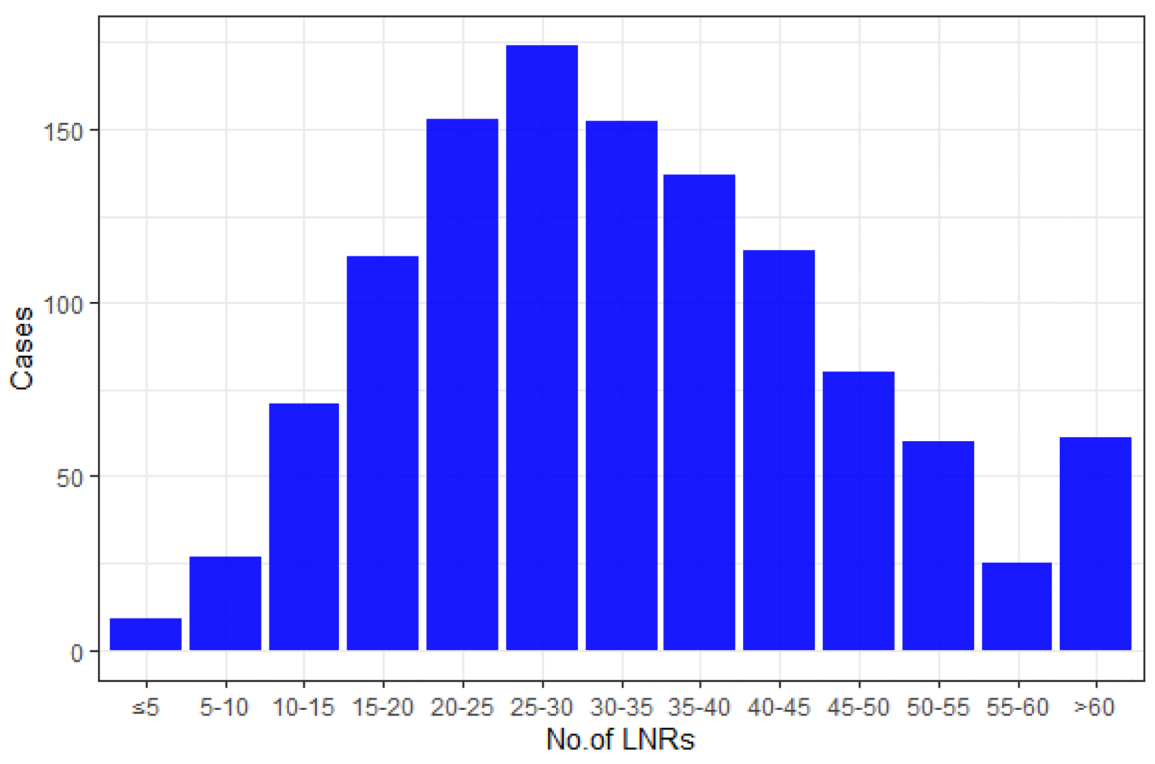

FIGURE 2 | The distribution of patients according to the number of LNRs. 


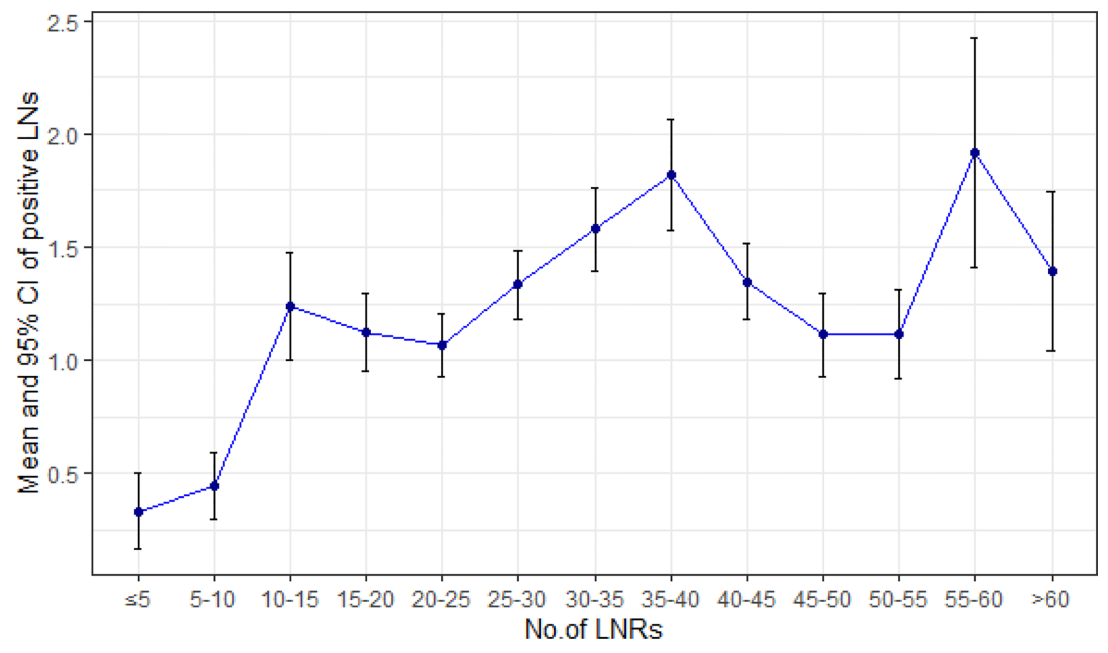

FIGURE 3 | Increasing pattern of mean numbers of positive LNs according to the number of LNRs.

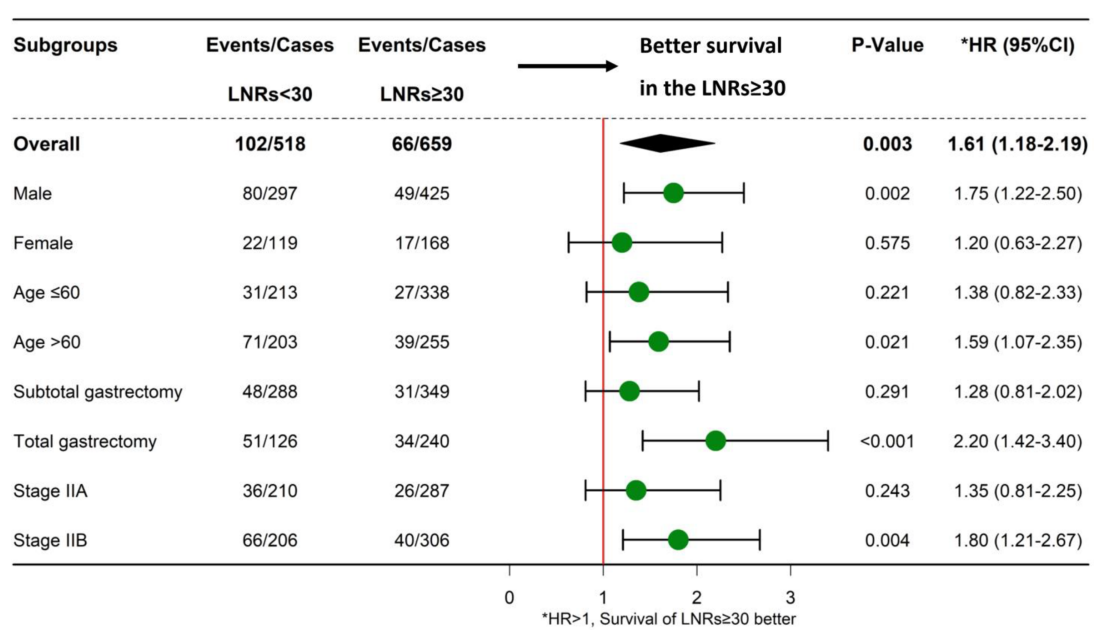

FIGURE 4 | Forest plot showing the impact of LNRs $(<30$ or $L N R s \geq 30)$ on survival in different subgroups.

increase in the number of LNRs. The impact of NR on survival is not statistically significant in the total sample, but in the subgroup analysis, NR is significantly correlated with worse survival in the $\mathrm{pN}+$ subgroup, especially for the $\mathrm{N}+$ patients with LNRs <30, as depicted in Figure 6.

\section{DISCUSSION}

According to the $8^{\text {th }}$ edition of the AJCC staging manual, retrieval of at least $16 \mathrm{LNs}$ is the minimal requirement after curative resection surgery $(14,15)$. However, gastric cancer is a malignancy with great heterogeneity, and applying the same standard to all patients in different conditions may lead to treatment bias. Previous studies have shown that increasing the
LNRs number is significantly correlated with prolonged survival for patients (3-10). Some researchers believe that retrieval of at least $16 \mathrm{LNs}$ is not sufficient to warrant adequate lymphadenectomy extension and accurate pathological staging. Retrieval of more LNs ( $>25$ or 30 ) is mandatory, while some researchers argue that retrieving more LNs only benefits those at more advanced stages (stage III), and attempting to retrieve 30 LNs for all patients is unnecessary. For example, Macalindong et al. found that the 5-year disease-free survival rate was not significantly different between the LNRs $<30$, LNRs 30-45, and LNRs $>45$ groups ( 72.9 vs 79.2 vs $76.2 \%$, $\mathrm{P}$ value $=0.566)$ in stage II patients (4). Vuong et al. also found that retrieving 30 or more LNs only resulted in a benefit for patients with pT1N3 and pT2N3 GC (11). Thus, the benefit of 30 LNRs is still controversial for stage II GC patients. 

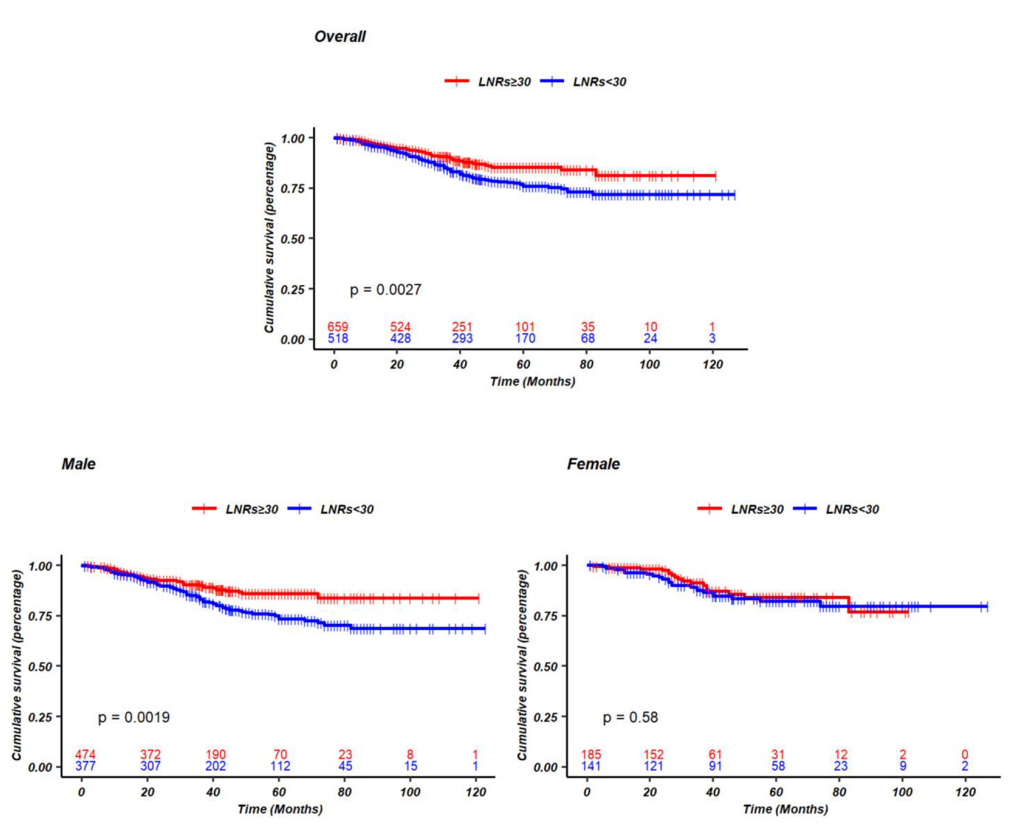

Age $\leq 60$

$$
+{ }_{L N R s \geq 30}+{ }_{L N R s<30}
$$

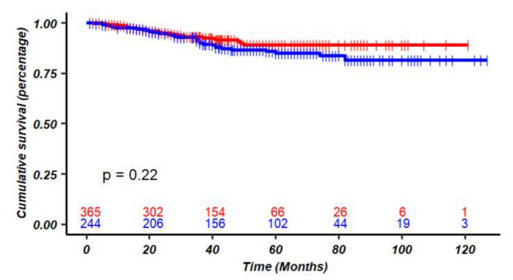

Total gastrectomy

$$
+{ }_{L N R S 230}+{ }_{L N R S<3 O}
$$

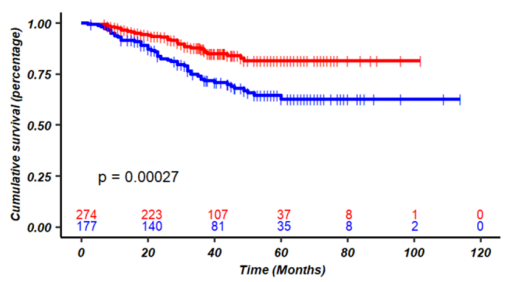

Stage IIA

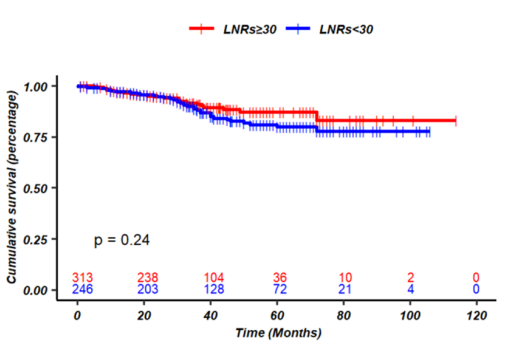

Age $>60$

$$
\text { - }{ }_{\text {LNRs }} 30+{ }_{\text {LNRs }}<30
$$

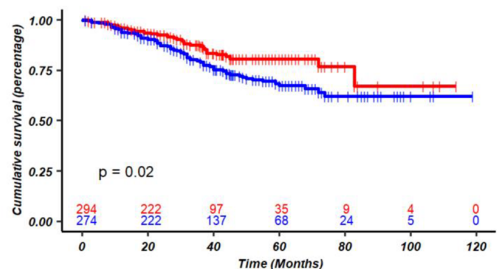

Subtotal gastrectomy

$$
+{ }_{L N R s \geq 30}+{ }_{L N R s<30}
$$

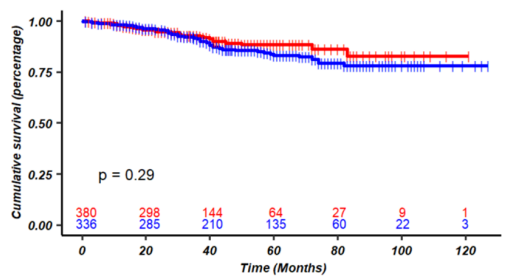

Stage IIB

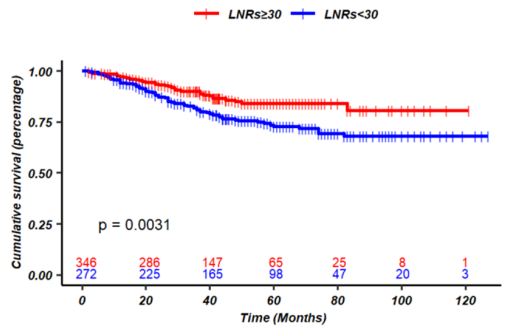

FIGURE 5 | Kaplan-Meier curves of survival comparing LNRs $<30$ and LNRs $\geq 30$ stratified by sex, age, extend of gastrectomy, different pTN stage. 

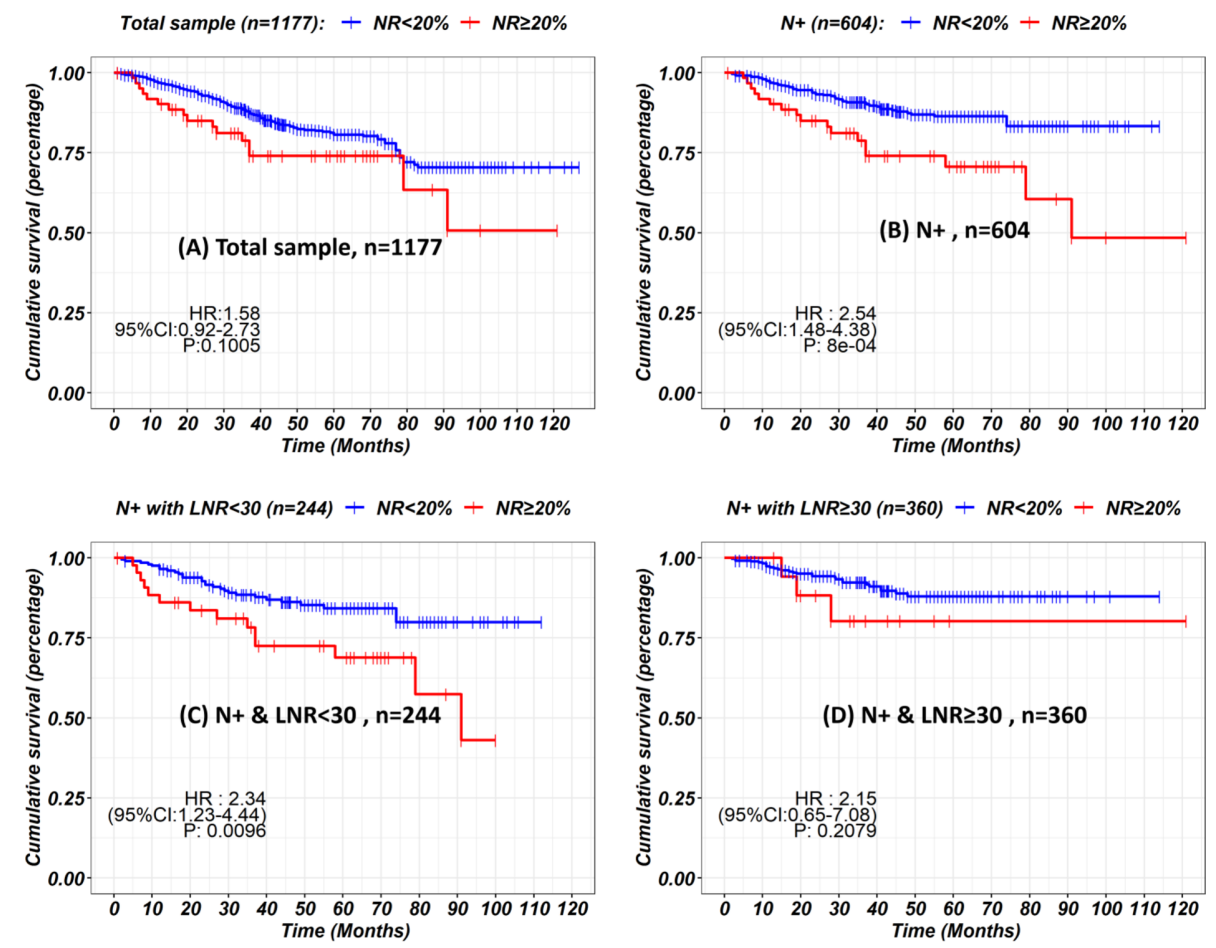

FIGURE 6 | Kaplan-Meier curves showing the impact of node ratio on survival in the total sample (A), N+ subgroups (B) stratified by LNRs (C, D).

In this study, we analyzed the survival impact of retrieving 30 or more LNs on the largest stage II gastric cancer cohort ever reported. A total of $88.7 \%(1,155 / 1,302)$ of patients had an LNRs $\geq 16$, and $53.4 \%(695 / 1,302)$ had an LNRs $\geq 30$, which is superior to previous reports, in which only $23-45 \%$ of patients had an LNRs $>16(5,16)$. Our finding is that retrieving 30 or more LNs is beneficial for stage II gastric cancer patients as a total group.

Increasing the number of LNRs benefits patients by providing a more accurate $\mathrm{N}$ stage and more extended lymphadenectomy. If hypothetically, we assume that the $\mathrm{N}$ stage is accurate when the number of LNRs is more than 16, then the mean number of positive LNs should remain steady when the LNRs are above 16. However, our data show that the mean number of positive LNs is still increasing when the LNRs are above 16, indicating that the $\mathrm{N}$ stage is still not reliable even if a minimum of 16 LNRs is met according to the AJCC staging manual. An insufficient LNRs will lead to stage migration, affecting the expected prognosis, sequential adjuvant therapy, and monitoring. Our data showed that the survival impact of NR was not significant in the total sample, but further subgroup analysis showed that higher NR was correlated with poor survival in $\mathrm{pN}+$ subgroup, especially in the $\mathrm{pN}+$ subgroup with LNRs $<30$. This could be explained by the constitution of stage II patients. As depicted in our data, approximately half of the stage II patients are with pN0 stage and a NR of $0 \%$, thus, the prognosis predictive value of NR is limited. But for the $\mathrm{pN}+$ patients with insufficient LNRs, introducing an index of positive lymph node ratio (NR) could potentially overcome the bias of inaccurate $\mathrm{N}$ staging, which is in concordance with the previous reports $(17,18)$. Nevertheless, the efforts by the surgeons and pathologists to retrieve more lymph nodes were important for avoiding false $\mathrm{pN}$ staging (19). To retrieve more lymph nodes, continuous cooperation between surgeons and pathologists is necessary. Surgeons should make their efforts to retrieve more lymph node during surgery following the standard of D2 regional lymphadenectomy (20). Adopting operative lymphatic tracer such as indocyanine green or carbon nanoparticles during lymphadenectomy had shown a great potential of increasing lymph node retrieval $(21,22)$. After surgery, it is highly recommended that the surgeons should handle the procedure of separating lymph node stations instantly on the fresh specimen, to improve LNRs and accuracy of station labeling (23-25).

Another important finding of our study is that the survival benefit of LNRs $\geq 30$ varies between different subgroups. For patients who underwent total gastrectomy, lymphadenectomy must be more extended according to the Japanese gastric cancer treatment guidelines (14). Thus, patients who receive total gastrectomy should undergo harvest of more LNRs than those who receive subtotal gastrectomy $(6,26)$. Bouvier et al. reported that the LNRs in total gastrectomy was higher than that of subtotal gastrectomy (10.4 in total gastrectomy; 7.2 in proximal gastrectomy; 7.4 in distal gastrectomy, $\mathrm{P}$ value $<0.0001$ ), which is in line with our findings (34.7 in total gastrectomy; 32.7 in subtotal gastrectomy, $\mathrm{P}$ value $=0.024)$. Patients with stage IIB 
disease were associated with a more advanced disease stage in the stage II group; thus, a more thorough and extended lymphadenectomy is warranted $(27,28)$, and an LNRs $\geq 30$ is mandatory. Additionally, an LNRs $\geq 30$ also benefits male/ age $>60$ elderly subgroups of patients.

There are a few limitations to our research. First, owing to the nature of the retrospective study design and the different origins of the data sets, treatment bias was inevitable. Second, the effect of confounding factors could not be eliminated in the subgroup analysis. Third, selection bias was also not neglectable because all patients enrolled were from three high-volume gastric cancer centers, and the high number of LNRs and ideal OS may not be easily reproducible in all centers.

\section{CONCLUSION}

Retrieval of 30 lymph nodes is mandatory for selected stage II gastric cancer patients.

\section{DATA AVAILABILITY STATEMENT}

The raw data supporting the conclusions of this article will be made available by the authors, without undue reservation.

\section{REFERENCES}

1. Sitarz R, Skierucha M, Mielko J, Offerhaus GJA, Maciejewski R, Polkowski WP. Gastric Cancer: Epidemiology, Prevention, Classification, and Treatment. Cancer Manag Res (2018) 10:239-48. doi: 10.2147/ CMAR.S149619

2. Amin MB, Greene FL, Edge SB, Compton CC, Gershenwald JE, Brookland RK, et al. The Eighth Edition AJCC Cancer Staging Manual: Continuing to Build a Bridge From a Population-Based to a More "Personalized" Approach to Cancer Staging. CA: Cancer J Clin (2017) 67(2):93-9. doi: 10.3322/ caac. 21388

3. Liu YY, Fang WL, Wang F, Hsu JT, Tsai CY, Liu KH, et al. Does a Higher Cutoff Value of Lymph Node Retrieval Substantially Improve Survival in Patients With Advanced Gastric Cancer?-Time to Embrace a New Digit. Oncologist (2017) 22(1):97-106. doi: 10.1634/ theoncologist.2016-0239

4. Macalindong SS, Kim KH, Nam BH, Ryu KW, Kubo N, Kim JY, et al. Effect of Total Number of Harvested Lymph Nodes on Survival Outcomes After Curative Resection for Gastric Adenocarcinoma: Findings From an Eastern High-Volume Gastric Cancer Center. BMC Cancer (2018) 18(1):73. doi: 10.1186/s12885-017-3872-6

5. Smith DD, Schwarz RR, Schwarz RE. Impact of Total Lymph Node Count on Staging and Survival After Gastrectomy for Gastric Cancer: Data From a Large US-population Database. J Clin Oncol Off J Am Soc Clin Oncol (2005) 23 (28):7114-24. doi: 10.1200/JCO.2005.14.621

6. Bouvier AM, Haas O, Piard F, Roignot P, Bonithon-Kopp C, Faivre J. How Many Nodes Must be Examined to Accurately Stage Gastric Carcinomas? Results Population Based Study Cancer (2002) 94(11):2862-6. doi: 10.1002/ cncr. 10550

7. Samples JE, Stitzenberg KB, Meyers MO. Lymph Node Yield and Survival in Gastric Carcinoma. J Clin Oncol (2014) 32(15_suppl):4012-4012. doi: 10.1200/jco.2014.32.15_suppl.4012

8. Huang CM, Lin JX, Zheng CH, Li P, Xie JW, Lin BJ. Effect of Negative Lymph Node Count on Survival for Gastric Cancer After Curative Distal

\section{ETHICS STATEMENT}

The studies involving human participants were reviewed and approved by the ethics committee of Sun Yat-Sen University Sixth Affiliated Hospital. The patients/participants provided their written informed consent to participate in this study. Written informed consent was obtained from the individual(s) for the publication of any potentially identifiable images or data included in this article.

\section{AUTHOR CONTRIBUTIONS}

J-SP and SC designed the study; Y-HC, JL and R-CN acquired the data; Y-HC analyzed, interpreted the data, and drafted the initial manuscript. DL made substantial revisions to the manuscript. A-HL, Z-JD, X-JC, JX, Y-BC, and C-MH helped with interpreting the data. Y-HC, JL, and R-CN contributed equally to this work.

\section{FUNDING}

Supported by Guangzhou Science and Technology Project (grant number 201803010040) and Nation Key Clinical Discipline.

Gastrectomy. Eur J Surg Oncol J Eur Soc Surg Oncol Br Assoc Surg Oncol (2011) 37(6):481-7. doi: 10.1016/j.ejso.2011.01.012

9. Kong SH, Lee HJ, Ahn HS, Kim JW, Kim WH, Lee KU, et al. Stage Migration Effect on Survival in Gastric Cancer Surgery With Extended Lymphadenectomy: The Reappraisal of Positive Lymph Node Ratio as a Proper N-Staging. Ann Surgery (2012) 255(1):50-8. doi: 10.1097/ SLA.0b013e31821d4d75

10. Karpeh MS, Leon L, Klimstra D , Brennan MF. Lymph Node Staging in Gastric Cancer: Is Location More Important Than Number? An analysis of 1,038 patients. Ann Surgery (2000) 232(3):362-71. doi: 10.1097/00000658200009000-00008

11. Vuong B, Graff-Baker AN, Dehal A, Stern S, Fujita M, Goldfarb M, et al. Survival Analysis With Extended Lymphadenectomy for Gastric Cancer: Removing Stage Migration From the Equation. Am Surg (2017) 83 (10):1074-9. doi: 10.1177/000313481708301012

12. Hartgrink HH, van de Velde CJ, Putter H, Bonenkamp JJ, Klein Kranenbarg E, Songun I, et al. Extended Lymph Node Dissection for Gastric Cancer: Who may Benefit? Final Results of the Randomized Dutch Gastric Cancer Group Trial. J Clin Oncol Off J Am Soc Clin Oncol (2004) 22(11):2069-77. doi: 10.1200/JCO.2004.08.026

13. Ajani JA, D'Amico TA, Almhanna K, Bentrem DJ, Chao J, Das P, et al. Gastric Cancer, Version 3.2016, NCCN Clinical Practice Guidelines in Oncology. J Natl Compr Cancer Netw JNCCN (2016) 14(10):1286-312. doi: 10.6004/ jnccn.2016.0137

14. Japanese Gastric Cancer A. Japanese Gastric Cancer Treatment Guidelines 2018 (5th Edition). Gastric Cancer (2021) 24(1):1-21. doi: 10.1007/s10120020-01042-y

15. Kim YI. Is Retrieval of At Least 15 Lymph Nodes Sufficient Recommendation in Early Gastric Cancer? Ann Surg Treat Res (2014) 87(4):180-4. doi: 10.4174/ astr.2014.87.4.180

16. Biondi A, D’Ugo D, Cananzi FC, Papa V, Borasi A, Sicoli F, et al. Does a Minimum Number of 16 Retrieved Nodes Affect Survival in Curatively Resected Gastric Cancer? Eur J Surg Oncol J Eur Soc Surg Oncol Br Assoc Surg Oncol (2015) 41(6):779-86. doi: 10.1016/j.ejso.2015.03.227 
17. Zhu J, Xue Z, Zhang S, Guo X, Zhai L, Shang S, et al. Integrated Analysis of the Prognostic Role of the Lymph Node Ratio in Node-Positive Gastric Cancer: A Meta-Analysis. Int J Surg (London England) (2018) 57:76-83. doi: 10.1016/ j.ijsu.2018.08.002

18. Wohnrath DR, Araujo RLC. Positive Node-Ratio in Curative-Intent Treatment for Gastric Cancer is a Strong Independent Prognostic Factor for 5-Year Overall Survival. J Surg Oncol (2020) 121(5):777-83. doi: 10.1002/ jso. 25755

19. Nienhüser H, Schmidt T. Gastric Cancer Lymph Node Resection-the More the Merrier? Trans Gastroenterol Hepatol (2018) 3:1. doi: 10.21037/ tgh.2017.12.08

20. Han SU, Hur H, Lee HJ, Cho GS, Kim MC, Park YK, et al. Surgeon Quality Control and Standardization of D2 Lymphadenectomy for Gastric Cancer: A Prospective Multicenter Observational Study (Klass-02-Qc). Ann Surgery (2021) 273(2):315-24. doi: 10.1097/SLA.0000000000003883

21. Chen QY, Xie JW, Zhong Q, Wang JB, Lin JX, Lu J, et al. Safety and Efficacy of Indocyanine Green Tracer-Guided Lymph Node Dissection During Laparoscopic Radical Gastrectomy in Patients With Gastric Cancer: A Randomized Clinical Trial. JAMA Surgery (2020) 155(4):300-11. doi: 10.1001/jamasurg.2019.6033

22. Li Z, Ao S, Bu Z, Wu A, Wu X, Shan F, et al. Clinical Study of Harvesting Lymph Nodes With Carbon Nanoparticles in Advanced Gastric Cancer: A Prospective Randomized Trial. World J Surg Oncol (2016) 14:88. doi: 10.1186/ s12957-016-0835-3

23. De Marco C, Biondi A, Ricci R. N Staging: The Role of the Pathologist. Trans Gastroenterol Hepatol (2017) 2:10. doi: 10.21037/tgh.2017.01.02

24. Davis PA, Sano T. The Difference in Gastric Cancer Between Japan, USA and Europe: What are the Facts? What are the Suggestions? Crit Rev Oncol/ hematol (2001) 40(1):77-94. doi: 10.1016/S1040-8428(00)00131-1
25. Catarci M, Montemurro LA, Di Cintio A, Ghinassi S, Coppola L, Pinnarelli L, et al. Lymph Node Retrieval and Examination During the Implementation of Extended Lymph Node Dissection for Gastric Cancer in a non-Specialized Western Institution. Updates Surgery (2010) 62(2):89-99. doi: 10.1007/ s13304-010-0017-8

26. Lu J, Wang W, Zheng CH, Fang C, Li P, Xie JW, et al. Influence of Total Lymph Node Count on Staging and Survival After Gastrectomy for Gastric Cancer: An Analysis From a Two-Institution Database in China. Ann Surg Oncol (2017) 24(2):486-93. doi: 10.1245/s10434-016-5494-7

27. Chen S, Nie RC, OuYang LY, Li YF, Xiang J, Zhou ZW, et al. Nomogram Analysis and External Validation to Predict the Risk of Lymph Node Metastasis in Gastric Cancer. Oncotarget (2017) 8(7):11380-8. doi: 10.18632/oncotarget.14535

28. Song W, Liu Y, Ye J, Peng J, He W, Chen J, et al. Proximal Gastric Cancer: Lymph Node Metastatic Patterns According to Different T Stages Dictate Surgical Approach. Chin Med J (Engl) (2014) 127(23):4049-54. doi: 10.3760/ cma.j.issn.0366-6999.20141566

Conflict of Interest: The authors declare that the research was conducted in the absence of any commercial or financial relationships that could be construed as a potential conflict of interest.

Copyright (C) 2021 Chen, Lu, Nie, Liu, Liu, Deng, Chen, Xiang, Chen, Huang, Chen and Peng. This is an open-access article distributed under the terms of the Creative Commons Attribution License (CC BY). The use, distribution or reproduction in other forums is permitted, provided the original author(s) and the copyright owner(s) are credited and that the original publication in this journal is cited, in accordance with accepted academic practice. No use, distribution or reproduction is permitted which does not comply with these terms. 